\title{
The Effect of Mucosidosis (Cystic Fibrosis) and Bacterial Factors on CFTR (CF Transmembrane Conductance Regulator) Channel Efficiency
}

\author{
Ali Ahmadi* \\ Department of Biological Sciences and Technologies, Islamic Azad University, Iran
}

\section{LETTER TO EDITOR}

Dear Editor,

Mucocidosis (cystic fibrosis) is the most common autosomal recessive inherited multisystem disease with a very deadly and dangerous outcome due to mutations and sequential mutations in the gene regulating the transmission of cystic fibrosis membrane (CFTR) leading to the chloride channel. And the CFTR protein becomes unhealthy and destructive [1,2]. As a result of the collection and collection of mucus that is not sufficiently hydrated and hydrophilic, it affects various basic organs, mainly lungs, and airways, pancreas, liver, bile ducts and intestines. This leads to inflammation and infection, fibrosis, and gradual tissue destruction [3-5]. Respiratory failure and subsequent problems have been cited as one of the leading causes of death. However, more than 30 years of molecular description A major defect in CFTR causes CF, a remarkable success in the long-term prognosis of people with CF. This breakthrough is very active in the spirit of collaboration and networking of the CF research community, and internationally, with the creation of a multidisciplinary clinical CF team that implements evidence in various aspects of standard care with the CF patient has been obtained. Rath et al. [5]. In their study found that several bacteria and fungi that cause organisms are also known as urinary tract infections (UTIs) that may occur in more than $50 \%$ of the population and are more common in women [5-8]. Urinary tract infection is one of the few and most limited bacterial infections approved by the laboratory, with an intrinsic limit of at least 3-4 days for the results of antibiotic susceptibility patterns to produce bacteria that cause bacteria in a urine sample. The experimental treatment is started according to the antibiotic inhibition program due to pain under radiation under the abdomen and inability to perform normal activities. This prescribed antibiotic often controls the disease. However, when there is an infection with a multidrugresistant bacterium (MDR), it is most often transmitted to cystitis and then to pyelonephritis $[8,9]$. Due to this problem, a clinician inadvertently prescribes an antibiotic with It prescribes a wide range, the generation, which insidiously considers the resistance to the same antibiotic in the world as a pathogen [10,11].

In another study, Chakotiya et al. [8] significantly increased the incidence of lung infections caused by Pseudomonas aeruginosa strains, which are classified as multidrug-resistant, mainly due to the relative inadequacy of potent chemotherapy. And is attributed to complication $[9,10]$. The present study was performed to evaluate the antimicrobial activity of the aqueous-alcoholic extract of Glycyrrhiza glabra against Paeruginosa causing lung infection in Swiss albino mice. This study included evaluating the lethal dose of $P$. aeruginosa in Swiss albino mice and analyzing disease manifestations that included bacteria, hypothermia, weight loss, and other parameters for 48 hours of infection. Physical manifestations of infected mice showed a significant decrease in body temperature from $29.582929{ }^{\circ} \mathrm{C}$ (in 48 hours) to 38.81 $33.33{ }^{\circ} \mathrm{C}(0 \mathrm{~h})$ and $30 \%$ weight loss at the end of the study. In addition, the effect of G. glabra extract against lung infection caused by lethal dose was calculated using bacteremia, histopathology, and radiological analysis. Bacterial load showed that 2.230-30 Log10CFU/ml on day 7 had a significant decrease in bacterial load compared to day 1 when Log10CFU/ml bacterial load decreased by 3.32 32.3. Histopathological results have shown that scattered and fragmented accumulation of inflammatory cells in the alveolar space has increased and also infiltrations have been observed in all parts of the lungs of infected mice $[5,8]$. In the treated animal's group, improved pulmonary histology with the discharge was less seen at D1 dose $(20 \mathrm{mg} / \mathrm{kg})$ and disappeared at D2 dose $(80 \mathrm{mg} /$ $\mathrm{kg}$ ). This study clearly states that G.glabra extract is effective against lung infection caused by P. aeruginosa at a dose of $80 \mathrm{mg} /$ kg. LCMS results showed that this extract contained glycyrrhizin, stigmasterol, and ergosterol, Liquocalcon, and guanidine. The present study is expected to further exploit the biomedical properties of this extract in preparing a strong diet against such threatening pathogens $[10,12,13]$.

\begin{tabular}{|l|l|} 
Quick Response Code: & $\begin{array}{l}\text { Address for correspondence: Ali Ahmadi, Department of Biological Sciences and } \\
\text { Technologies, Islamic Azad University, Iran }\end{array}$ \\
& $\begin{array}{l}\text { Received: October 14, } 2021 \quad \text { Published: October 27, } 2021 \\
\text { How to cite this article: Ali A. The Effect of Mucosidosis (Cystic Fibrosis) and Bacterial Factors } \\
\text { on CFTR (CF Transmembrane Conductance Regulator) Channel Efficiency. 2021- 3(5) OAJBS. } \\
\text { ID.000342. DOI: } 10.38125 / O A J B S .000342\end{array}$ \\
\hline
\end{tabular}


The resistance of multi drugs presented and evaluated by Pseudomonas aeruginosa to antibiotics was also reflected in its tendency to produce biofilms, cell membrane modifications, and antibacterial drugs. The study of the activity of Glycyrrhiza glabra and one of its pure compounds, glycyrrhizic acid against $P$. aeruginosa and their mechanism of action in terms of the effect on membrane permeability, flow activity, and biofilm formation has been determined [14,15]. Minimum inhibitory concentration with the dilution method and the amount of dilution of broth in the casting medium has been determined [16,17]. The minimum antibacterial concentration on the agar plate has been investigated The MIC of the extract and glycyrrhizic acid were 200 and $100 \mu \mathrm{gml}$ $(-1)$, respectively. The MBC for the extract and glycyrrhizic acid is 800 and $400 \mu \mathrm{g}$, respectively. The effect of time-dependent homicide has also been estimated. Flow cytometric analysis was performed to determine the effect of extract and glycyrrhizic acid at $2 \times$ MIC on various physiological parameters using staining methods and comparison with a standard (antibiotic). The growth of Paeruginosa was significantly inhibited by extracts and pure compounds. Plant extracts and glyceric acid were also effective in targeting the physiological parameters of bacteria, including cell membrane permeability, flow activity, and biofilm formation. This study reports the anti-pseudomonal function of Glycyrrhiza glabra and one of its compounds and provides insight into their function $[8,17,10]$.

\section{REFERENCES}

1. Ringshausen F, Hellmuth T, Dittrich A (2020) Evidence-based treatment of cystic fibrosis. Der Internist 61(12): 1212-1229.

2. Muraglia KA, Rajeev SC, Bo Ram K, Xiao XT, Viral SS, et al. (2019) Smallmolecule ion channels increase host defenses in cystic fibrosis airway epithelia. Nature 567(7748): 405-408

3. Reznikov LR, Mahmoud HA, Cassie LD, Nick DG, Daniel JD, et al. (2014) Antibacterial properties of the CFTR potentiator ivacaftor. J Cyst Fibros 13(5): 515-519.

4. Sandri A, Lleo MM, Signoretto C, Boaretti M, Boschi F (2021) Protease inhibitors elicit anti-inflammatory effects in CF mice with Pseudomonas aeruginosa acute lung infection. Clin Exp Immunol 203(1): 87-95.

5. Rath S, Padhy RN (2014) Monitoring in vitro antibacterial efficacy of 26 Indian spices against multidrug resistant urinary tract infecting bacteria. Integrative Medicine Research 3(3): 133-141.
6. Anand S, Mandal S, Patil P, Tomar SK (2016) Pathogen-induced secretory diarrhea and its prevention. Eur J Clin Microbiol Infect Dis 35(11): 17211739.

7. Sofoluwe A, Alice Z, Marc B, Sylvain L, Marc C (2020) Immune response of polarized cystic fibrosis airway epithelial cells infected with Influenza A virus. J Cyst Fibros 20(4): 655-663.

8. Chakotiya AS, Ankit T, Alka N, Rakesh KS, Pranay S (2017) Effect of aquoalchoholic extract of Glycyrrhiza glabra against Pseudomonas aeruginosa in Mice Lung Infection Model. Biomed Pharmacother 90: 171-178.

9. Chakotiya, AS, Ankit T, Alka N, Rakesh KS (2016) Alternative to antibiotics against Pseudomonas aeruginosa: Effects of Glycyrrhiza glabra on membrane permeability and inhibition of efflux activity and biofilm formation in Pseudomonas aeruginosa and it's in vitro time-kill activity. Microb Pathog 98: 98-105.

10. Chakotiya AS, Raman C, Pallavi T, Ankit T, Alka N, et al. (2016) In vitro bactericidal activity of promising nutraceuticals for targeting multidrug resistant Pseudomonas aeruginosa. Nutrition 32(7-8): 890-897.

11. Lorentzen D, Lakshmi D, Alejandro AP, Yoko N, Janice L, et al. (2011) Concentration of the antibacterial precursor thiocyanate in cystic fibrosis airway secretions. Free Radic Biol Med 50(9): 1144-1150.

12. Di Pietro C, Ping-Xia Z, Timothy K, Thomas SM, Lin W, et al. (2017) Ezrin links CFTR to TLR4 signaling to orchestrate anti-bacterial immune response in macrophages. Sci Rep 7(1): 10882.

13. Brockman SM, Manish B, David S, Ajit S, Neeraj V (2017) Dendrimerbased selective autophagy-induction rescues $\Delta$ F508-CFTR and inhibits Pseudomonas aeruginosa infection in cystic fibrosis. PLoS One 12(9): e0184793.

14. Zhou YM, Lei S, Ji-an L, Li-zhong H, Wai-jiao C, et al. (2011) An efficient and novel screening model for assessing the bioactivity of extracts against multidrug-resistant Pseudomonas aeruginosa using Caenorhabditis elegans. Biosci Biotechnol Biochem 75(9): 1746-1751.

15. Rahman H, Ilyas K, Anwar H, Abdelaaty AS, Abdul T, et al. (2018) Glycyrrhiza glabra HPLC fractions: identification of Aldehydo Isoophiopogonone and Liquirtigenin having activity against multidrug resistant bacteria. BMC Complement Altern Med 18(1): 140.

16. Marteyn BS, Pierre-Régis B, Laurent M, Véronique W (2017) Harnessing neutrophil survival mechanisms during chronic infection by Pseudomonas aeruginosa: Novel therapeutic targets to dampen inflammation in cystic fibrosis. Front Cell Infect Microbiol 7: 243.

17. Chakotiya AS, Ankit T, Alka N, Rakesh K (2017) Zingiber officinale: Its antibacterial activity on Pseudomonas aeruginosa and mode of action evaluated by flow cytometry. Microb Pathog 107: 254-260. 